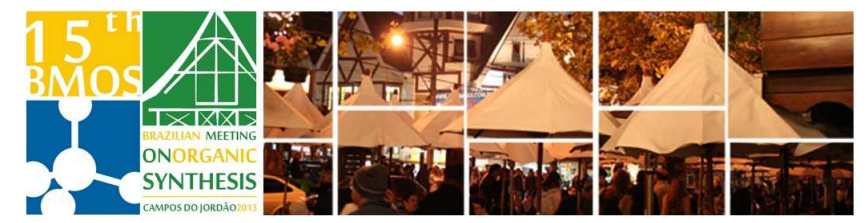

\title{
Synthesis of Six-membered Ring Analogues of RK-682 and Meldrum's acid derivatives. Evaluation on the Inhibition of Protein Tyrosine Phosphatases
}

\author{
Vânia M. T. Carneiro, Daniela B. B. Trivella, Ricardo Aparicio and Ronaldo A. Pilli* \\ Chemistry Institute, University of Campinas - UNICAMP, CP 6154, CEP 13083-970, Campinas SP, Brazil. \\ *pill@iqm.unicamp.br
}

Keywords: RK-682, Meldrum's acid, phosphatases

\section{INTRODUCTION}

Protein tyrosine phosphatases (PTPs) together with protein tyrosine kinases (PTKs) regulate protein tyrosine phosphorylation. Their abnormal functions are involved in the initiation and maintenance of the oncogenic state in human. ${ }^{1}$ Several synthetic tetronic acid derivatives related to the natural product RK-682 $(R)$-1 (Figure 1) have been evaluated as inhibitors of dual-specificity phosphatases VHR and CDC-25B and four derivatives were shown to be the most potent inhibitors of CDC-25B described so far. ${ }^{2}$

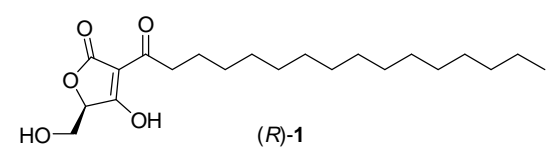

Figure 1. Natural product RK-682.

The scope of this work includes the synthesis of sixmembered ring homologues of RK-682 and Meldrum's acid derivatives and evaluation of their inhibition against human protein tyrosine phosphatases (LMW-PTP, CDC-25B and PTP-1B).

\section{RESULTS AND DISCUSSION}

Ethyl 4-chloroacetoacetate was converted to intermediate 5 after 7 steps. Synthesis of sixmembered ring homologues 6 and 7 was carried out via coupling between $\mathbf{5}$ and palmitic acid mediated by $N, N$-dicyclohexylcarbodiimide (DCC) (Scheme 1).

Scheme 1. Synthesis of 6 and 7.

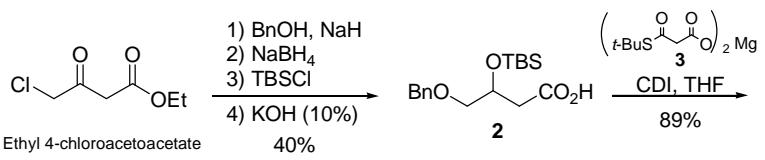

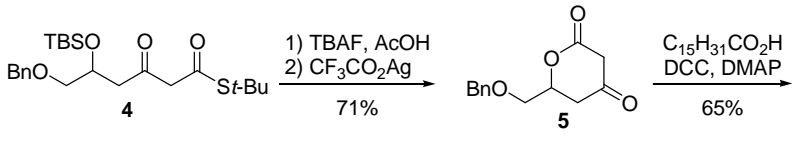

$$
\begin{aligned}
& \underbrace{\left.\mathrm{CH}_{2}\right)_{14} \mathrm{CH}_{3}}_{\mathrm{OH}} \underset{69 \%}{\stackrel{\mathrm{Pd}-\mathrm{C}(20 \% \mathrm{w} / \mathrm{w})}{\mathrm{Et}_{3} \mathrm{SiH}, \mathrm{EtOH}}}
\end{aligned}
$$$$
\text { Ethyl 4-chloroacetoacetate } \quad 40 \%
$$

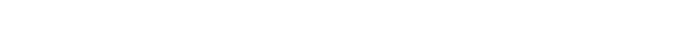

Employing a similar DCC-mediated coupling, ${ }^{3}$ compounds 8-16 were prepared from Meldrum's acid derivatives (Scheme 2).

Scheme 2. Synthesis of dioxinones.

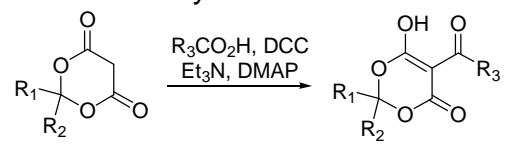

Meldrum's acids

8, $\mathrm{R}_{1}=\mathrm{R}_{2}=\mathrm{CH}_{3}, \mathrm{R}_{3}=\left(\mathrm{CH}_{2}\right){ }_{14} \mathrm{CH}_{3}, 79 \%$ 9, $R_{1}=R_{2}=C_{3}, R_{3}=\left(C_{2}\right)_{16} C_{3}, 85 \%$

10, $\mathrm{R}_{1}=\mathrm{R}_{2}=\mathrm{CH}_{3}, \mathrm{R}_{3}=\left(\mathrm{CH}_{2}\right)_{8} \mathrm{CH}_{3}, 65 \%$

$\begin{array}{ll}10, R_{1} & =R_{2}=C_{3}, R_{3}=\left(\mathrm{CH}_{2}\right)_{8} \mathrm{CH}_{3}, 65 \% \\ 11, R_{1}=\mathrm{R}_{2}=\mathrm{CH}_{3}, \mathrm{R}_{3}=\mathrm{CH}\left(\mathrm{CH}_{3}\right)_{2}, 40 \%\end{array}$

$12, \mathrm{R}_{1}=\mathrm{R}_{2}=\mathrm{CH}_{3}, \mathrm{R}_{3}=\left(\mathrm{CH}_{2}\right)_{2} \mathrm{CH}_{3}, 55 \%$

13, $R_{1}=\mathrm{R}_{2}=\mathrm{CH}_{3}, \mathrm{R}_{3}=\mathrm{CH}_{3}, 69 \%$

14, $\mathrm{R}_{1}=\mathrm{CH}_{3}, \mathrm{R}_{2}=\mathrm{CH}_{2} \mathrm{CH}_{3}, \mathrm{R}_{3}=\left(\mathrm{CH}_{2}\right){ }_{14} \mathrm{CH}_{3}, 53 \%$

15, $\mathrm{R}_{1}=\mathrm{R}_{2}=\mathrm{CH}_{2} \mathrm{CH}_{3}, \mathrm{R}_{3}=\left(\mathrm{CH}_{2}\right){ }_{14} \mathrm{CH}_{3}, 60 \%$

16, $\mathrm{R}_{1}=\mathrm{R}_{2}=\mathrm{CH}_{2} \mathrm{CH}_{2}, \mathrm{R}_{3}=\left(\mathrm{CH}_{2}\right){ }_{14} \mathrm{CH}_{3}, 38 \%$

The screening of their PTP inhibitory activity and comparison with racemic RK-682 (rac-1) has shown that the six-membered compounds 6 and 7 were inactive in the LMW-PTP inhibition assays, displayed reduced PTP-1B inhibitory activity but kept similar activity against CDC-25B. In contrast, dioxinanes $\mathbf{8}$ and $\mathbf{9}$ showed potent inhibitory activity for the three phosphatases analyzed (Table 1).

Table 1. Inhibition of LMW-PTP, CDC-25B and PTP-1B

\begin{tabular}{cccc}
\hline & & $\mathrm{IC}_{50}(\mu \mathrm{M})$ & \\
\hline & LMW-PTP & CDC-25B & PTP-1B \\
\hline rac-1 & $8.62 \pm 2.98$ & $12.43 \pm 1.83$ & $0.51 \pm 0.01$ \\
$\mathbf{6}$ & $>100$ & $3.45 \pm 4.04$ & $5.63 \pm 0.76$ \\
$\mathbf{7}$ & $>100$ & $13.63 \pm 3.73$ & $15.58 \pm 0.23$ \\
$\mathbf{8}$ & $20.02 \pm 2.62$ & $13.65 \pm 2.34$ & $1.08 \pm 0.00$ \\
$\mathbf{9}$ & $5.31 \pm 0.35$ & $6.50 \pm 0.23$ & $0.56 \pm 0.11$ \\
\hline
\end{tabular}

\section{CONCLUSION}

Compounds 6 and 7 displayed greater selectivity to PTP-1B and CDC-25B than racemic RK-682. Dioxinones 8 and 9 have shown similar PTPase inhibitory activity as the natural compound RK-682.

\section{ACKNOWLEDGEMENTS \\ FAPESP (2009/51602-5, 2010/17545-5 and 11/00457-5).}

\section{REFERENCES}

${ }^{1}$ Scott, M. L. et al. Curr. Pharm. Des. 2010, 16, 1843.

${ }^{2}$ Sodeoka, M. et al. J. Med. Chem. 2001, 44, 3216.

${ }^{3}$ Pashkovskii, F. S. et al. Russ. J. Org. Chem. 2003, 39, 1060. 